Studi

\title{
Wissenskulturen, Wissensgeschichte und historische Epistemologie
}

Claus Zittel

Ricevuto il 14 marzo 2013; accettato il 23 settembre 2013

Abstract Cultures of Knowledge, History of Knowledge, and Historical Epistemology - The term culture of knowledge has become a commonplace today and is circulating with a confusing diversity of meanings throughout various disciplines and fields, like Cultural Studies, Science Studies, History of Science, Epistemology, Sociology and History of Knowledge. This paper investigates the origins of the term culture of knowledge and discusses its various meanings, applications, and misconceptions in Cultural Theory, History of Science, Historical Epistemology and History of Knowledge. Finally, Ludwik Fleck's theory of thought-styles is proposed as an ideal model for describing specific cultures ofknowledge.

KEYwORDS: Cultures of Knowledge; Epistemic Contextualism; Social Epistemology; Relativism; History and Philosophy of Science; Ludwik Fleck.

Riassunto Culture epistemiche, storia del sapere ed epistemologia storica - Il termine "cultura epistemica" è oggi diventato un luogo comune e sta attraversando, con una diversità di significato che genera confusione, varie discipline e aree del sapere, come gli studi culturali, le ricerche scientifiche, la storia della scienza, la sociologia e la storia della conoscenza. Questo lavoro indaga le origini del termine "cultura epistemica" e discute i suoi diversi significati, le sue diverse applicazioni e i vari fraintendimenti cui è andato incontro nella teoria della cultura, nella storia della scienza, nell'epistemologia storia e nella storia della conoscenza. In conclusione, la teoria degli stili di pensiero di Ludwik Fleck sarà proposta come modello ideale per descrivere le culture epistemiche nella loro specificità.

PAROLE CHIAVE: Culture epistemiche; Contestualismo epistemico; Epistemologia sociale; Relativismo; Storia e filosofia della scienza; Ludwik Fleck.

\section{番}

Das Können, nicht das Wissen, durch die Wissenschaft geübt. F. NIETZSCHE, Menschliches, Allzumenschliches

\section{Wissenskulturen}

»IST NICHT JEDE KULTUR EINE WISSENSKULTUR?« fragte mich im Jahr 1999 erstaunt ein Kollege, nachdem er zufällig das Schild an der Tür meines frisch bezogenen Büros an der Goethe-Universität zu Frankfurt entdeckt hatte: >SFB/FK 435 - Wissenskultur und gesellschaftlicher Wandel<. Dass der Begriff $>$ Wissenskultur < noch vor 14 Jahren Irritation auslöste, ist heute vergessen. Großzügig werden mittlerweile alle Arten von Kulturen zu Wissenskulturen erklärt, nur die Bakterienkulturen

C. Zittel - Institut für deutsche und niederländische Philologie - Freie Universität Berlin - Habelschwerdter Allee 45 - 14195 Berlin (D) ( $\square)$

E-mail: zittel@zedat.fu-berlin.de 
leisten noch letzten Widerstand.

Der Terminus $>$ Wissenskultur $<$ gleitet nun vielen leicht und schnell über die Zunge, und dies nicht nur, um Forschungskulturen in den Naturwissenschaften $\mathrm{zu}$ bezeichnen. Der Trend manifestiert sich auch darin, dass vorzugsweise in den Geisteswissenschaften immer mehr Vorlesungsreihen, Professuren und Masterstudiengänge den Wissenskulturen gewidmet werden. ${ }^{1}$ Unterdessen untersucht man die fremden Wissenskulturen der Osmanen ${ }^{2}$ und der Irokesen, ${ }^{3}$ die besonderen des Designs ${ }^{4}$ und des Tanzes, ${ }^{5}$ - ja es ist sogar möglich von der »Wissenskultur und Poetik der Transplantationsmedizin $\ll,{ }^{6}$ von $\gg$ religiösen Wissenskulturen $\ll^{7}$ oder von »abergläubischen Wissenskulturen der Dämonologie $\ll^{8}$ zu sprechen, ohne Gefahr zu laufen, sofort des Widersinns überführt zu werden.

Mit dem Siegeszug des Begriffs verblasste zugleich die Erinnerung an die einfachen Fragen, die er anfangs noch provozierte: Wie ist das Verhältnis von Kultur und Wissen? Welchen Begriff von Kultur und von Wissen setzt man jeweils voraus, sobald man das Kompositum $>$ Wissenskultur $<$ bildet? Wie umfangreich ist eine Wissenskultur vorzustellen? Denken wir hier in großen Dimensionen wie die Wissenskultur der Antike, des Abendlands oder Asiens, oder nehmen wir eher kleinere Einheiten z.B. wie die Wissenskultur der TocharischExperten oder der analytischen Philosophie in den Blick? Sind nur Hoch- oder Expertenkulturen Wissenskulturen oder auch Alltagskulturen? Sind diese Kulturen an Institutionen oder zumindest an Schrift gebunden oder gibt es auch orale Wissenskulturen? Und vor allem: Warum und mit welchen Folgen bezeichnen wir Kulturen als Wissenskulturen? Wie stark relativiert die Rückbindung des Wissensbegriffs an die Kultur denselben und wie weit oder eng also ist er zu fassen?

Man kann angesichts des allgemeinen unbekümmerten Gebrauchs des Wissenskulturbegriffs sich nun dieser Entwicklung entweder entgegenstellen oder sie als Phänomen studieren. Im ersten Falle wählt man den in der Philosophie vertrauten Weg, zuerst einmal zu ver- suchen, den kulturwissenschaftlichen und alltagssprachlichen Wildwuchs des Begriffes zurückzustutzen, indem man die Implikationen des Terminus $>$ Wissenskultur $<$ bewusst macht und verdeutlicht, welche Begriffe von Wissen und Kultur in der jeweiligen Verwendungsweise vorausgesetzt werden. In diesem Fall übernimmt man die Rolle des ungeliebten Linienrichters, der den anderen Akteuren jeweils erklärt, dass sie eigentlich den Begriff so nicht verwenden dürfen oder diese oder jene Konsequenz akzeptieren müssen. Das Spiel ist jedoch bereits so chaotisch geworden, dass die Verteidiger von Regeln hier auf verlorenen Posten stehen. Die zweite Variante wäre, die Vielfalt und Buntheit der gegenwärtig zirkulierenden Verwendungsweisen mit dem Begriff selbst in Verbindung zu bringen. Denn die Omnipräsenz des Ausdrucks > Wissenskultur< indiziert schlicht, dass offensichtlich der Begriff des Wissens in den vergangenen Jahren radikal historisiert, sozial und kulturell relativiert und in seinem unhintergehbaren standpunktgebundenen Konstruktcharakter durchschaut wurde.

Im Folgenden werde ich daher darauf verzichten, dem Phantom einer Begriffsdefinition nachzujagen, sondern funktionsanalytisch untersuchen, wann und wo der Begriff der Wissenskultur auftauchte, wie er gebraucht wurde, und überlegen, wie man ihn für die Historiographie des Wissens sinnvoll eingrenzen und einsetzen könnte.

\section{Zur Geschichte des Begriffs 'Wissenskultur‘}

Offenkundig war der DFG-geförderte Sonderforschungsbereich 435 an der Frankfurter Goethe-Universität: Wissenskultur und gesellschaftlicher Wandel, in dessen zehnjähriger Laufzeit 38 Monographien und Sammelbände in der gleichnamigen Reihe erschienen, maßgeblich an der Verbreitung und Durchsetzung des Begriffs im deutschsprachigen Raum beteiligt. Eine wichtige Rolle spielte auch das 2002 auf deutsch unter dem Titel Wissenskulturen erschienene Buch von Karin Knorr Cetina. ${ }^{9}$ Nun ist - wie bereits der Untertitel ausweist Knorr Cetinas notorisch auch seitens der Kul- 
turwissenschaften als einschlägig angeführtes Buch ausdrücklich dem $\gg$ Vergleich naturwissenschaftlicher Wissensformen« gewidmet, den Expertenkulturen in den Naturwissenschaftskulturen also, und deren verschiedenen Praktiken der Wissenserzeugung.

Der SFB/FK 435 jedoch war seinerzeit das größte geisteswissenschaftliche Forschungskolleg unter Beteiligung von 13 verschiedenen Disziplinen; sein Begriff der Wissenskultur war also von Anfang an breiter und historisch angelegt. Der Untertitel zum Programmband der Reihe Wissenskultur verspricht daher bezeichnenderweise >Beiträge $\mathrm{zu}$ einem forschungsstrategischen Konzept $<.{ }^{10}$ Es ging vordringlich darum, eine transdisziplinäre Fragestellung zu formulieren und die unterschiedlichen disziplinären Modifikationen der Frage und spezifischen Antworten vergleichend zu betrachten. $\mathrm{Zu}$ einem einheitlichen Verständnis, was Wissenskulturen sind, ist man nie gelangt, und dies war auch nicht das Ziel.

Paradoxerweise verleiht der Begriff der $>$ Wissenskulturen $<$ dem geisteswissenschaftlichen Wissen einen Hauch naturwissenschaftlicher >Objektivität<, während er zugleich dazu tendiert, den Wissensbegriff und damit die Objektivitätsansprüche sogar der Naturwissenschaften aufzulösen. Denn mit der Wahl wissenskultureller Ansätze geht zwangsläufig eine Relativierung des Wissensbegriffs einher, die so weitgehend ist, dass die Wahrheitsbedingung für Wissensansprüche aufgegeben und die Rechtfertigungsbedingung nicht mehr ausschließlich auf Begründungskontexte beschränkt werden kann. Der Begriff der Wissenskultur verschleiert somit erfolgreich, dass mit ihm skeptische Positionen befördert werden, weshalb unter seiner Tarnkappe bunt-heterogene und relativistische Ansätze auch in die Forschungsförderung, den öffentlichen Wissensdiskurs und die universitäre Lehre sich einschmuggeln konnten. Das muss man nicht beklagen.

Auch wenn zuweilen von Mitstreitern der Anspruch erhoben wurde, das Frankfurter Forschungskolleg habe den Begriff $>$ Wissenskultur $<$ geprägt, ${ }^{11}$ so ist zwar zu konzedieren, dass vor 1999 der Terminus eher selten und mit ei- ner gleich noch zu nennenden Ausnahme unspezifisch begegnete, der Sache nach als heuristisches Konzept jedoch durchaus bereits länger präsent war. Jedenfalls entbehrt seine Begriffsgeschichte nicht einiger verblüffender Volten und Kapriolen.

So resümierte Bernhard Dotzler ${ }^{12}$ in einem auch den Zeitraum vor 1999 auslotenden Forschungsbericht: »Unter den kulturellen Selbstbeschreibungen der Gegenwart hat ihre Definition als Wissenskultur zunehmend an Plausibilität gewonnen $\ll{ }^{13}$ Dotzler verweist auf die Entwicklungen in den Science Studies, denen sich auch Knorr Cetinas Buch verdankt, in welchen literatur- und kulturwissenschaftliche Begriffe, Metaphern und Methoden in die Wissenschaftsforschung Einzug gehalten hätten und sich die Wissenschaftsgeschichte stärker für die Kulturgeschichte geöffnet habe. In der Tat zeigen die im Zuge des sogenannten cultural turns aufgekommenen Studien zur Rhetorik der Wissenschaft, zu literarischen Technologien, Diskursen und Narrativen in den Wissenschaften, ihren Repräsentationsformen und Metaphern solche Übernahmen an.

Unter dem Namen der Wissenskultur vollzieht sich dann letztlich nur ein Re-Import der alten kulturwissenschaftlichen Konzepte, die mittlerweile von der Wissenschaftsforschung nobilitiert, Seriösität und Antragstauglichkeit gewonnen haben, vor allem weil sie als neues Forschungsfeld die Verbindung von Literatur und Naturwissenschaften vorzeigen können.

Die Geschichte wird jedoch komplizierter, je tiefer man gräbt. Der erste prominente Beleg für den Gebrauch des Terminus >Wissenskultur< findet sich in einem klassischen Text der Kulturtheorie, in Nietzsches Frühschrift Die Geburt der Tragödie aus dem Geiste der Musik. Nietzsche beschreibt hierin die Verfallsgeschichte der griechischen Kultur, die nur solange sie sich vom Mythos speiste, lebendig und schöpferisch geblieben sei. Durch den Siegeszug des Sokratismus sei jedoch eine der beiden mythischen Grundkräfte, das Prinzip des Apollinischen, hypertrophiert und zu einseitiger Dominanz über das dionysische Prinzip gelangt und die Selbstzerstörung des Griechentums unaufhaltsam ge- 
worden. Triebkraft sei der sokratische »unumschränkt sich wähnende Optimismus «, der in der Wahnvorstellung, alles erklären zu können sowie im »Glaube[n] an das Erdenglück aller « bestünde. Zur Katastrophe käme es, wenn der Glaube »an die Möglichkeit einer solchen allgemeinen Wissenscultur allmählich in die drohende Forderung eines solchen alexandrinischen Erdenglückes [...] umschlägt! $\ll .{ }^{14}$

Nicht jede Kultur also ist für Nietzsche eine Wissenskultur, im Gegenteil: Der Begriff $>$ Wissenskultur< wird von ihm klar negativ besetzt, er ist fast ein Oxymoron und eigentlich ein $\mathrm{Pa}$ radox, da er Nicht-Zusammengehörendes zusammenzwingt. In Nietzsches Augen ist in dem Augenblick, wo eine Kultur zur Wissenskultur wird, sie dem Untergang geweiht, da sie ihre schöpferischen Wurzeln kappt und ihre irrationalen Ursprünge verleugnet. Sie degeneriert. Dabei ist die sokratische Wissenskultur nicht als dem Mythos einfach entgegensetzt gedacht, mithin nicht als Kultur sui generis, sondern als die äußerste Verdichtung des Mythos begriffen. Das Fatale an einer Wissenskultur ist also nicht nur, dass sie sich gegen den Mythos stellt, sondern dass sie nicht erkennt, selbst vollkommen von irrationalen Mächten und Wahnvorstellungen beherrscht zu sein.

Die Repräsentanten der Wissenskultur befinden sich in einer zwanghaften Selbsttäuschung, wenn sie glauben, sie hätten den Mythos überwunden, dessen tragischer Impetus gerade in der durch den Sokratismus besorgten Zerstörung der griechischen Kultur Triumphe feiert. In diesem Lichte erscheinen die heutigen Versuche von Nietzsches kulturphilosophischen Urenkeln, ästhetische Kulturen aufzuwerten, ausgerechnet indem sie Kunst als Wissensform, künstlerische Praktiken als Forschung beschreiben und Künstler zu Wissenschaftler küren, als ein Bärendienst an der Kultur, gleichsam als finaler Rettungsschuss des Sokratismus.

Zunächst noch in seiner kulturkritischen Fassung indes wanderte der Begriff der Wissenskultur in den 1920er Jahren in die Wissenssoziologie ein. Max Scheler warnt in seiner Abhandlung: Wissensformen der Gesellschaft beispielsweise in bezeichnender Zusammenstel- lung vor der > grosse[n] Gefahr für die >abendländische Wissenskultur< wie der positivistische Szientifismus, die marxistische Einbildung einer > proletarische Wissenschaft $<$ und die vordringenden kirchlichen Scholastiken mit ihren engen kleinen Trutz- und Schutzbauten gegen den Strom der Zeit $\ll .{ }^{15}$ Scheler kennt offenbar die Möglichkeit einer - allerdings global und im Singular vorgestellten - Wissenskultur, sieht diese aber gerade inter alia durch eine zu starke Dominanz der Naturwissenschaften bedroht. >Kulturlose Wissenschaft contra abendländische Wissenskultur< wäre eine Formel für seine Position.

Nun figuriert neben der Wissenssoziologie auch die historische Epistemologie häufig als theoretische Vorläufer-Instanz für Wissenskulturkonzepte. In spiegelbildlicher Entsprechung zu Schelers Position hatten historische Epistemologen wie Bachelard ihre Aufgabe gerade darin gesehen, das wissenschaftliche Wissen von kulturellen Wissenformen zu scheiden und sich dabei primär auf die Validierung wissenschaftlichen Wissens konzentriert (s.u.). Auch die aus der historischen Epistemologie hervorgegangene Diskursanalyse, bei der ebenfalls häufig methodische Anleihen gemacht werden, um Wissenskulturen $\mathrm{zu}$ beschreiben, ist eine problematische Quelle, insofern sich Diskurse primär auf Aussagen und den Relationen zwischen denselben beziehen, die unbewussten Dynamiken, das tacit knowing, oder auch die visuellen Praktiken der Evidenzerzeugung beispielsweise durch Bilder, die allesamt Wissenskulturen heute als Charakteristika zugeschrieben werden, methodisch aber gerade nicht einfangen. ${ }^{16}$ Darauf komme ich noch zurück.

Der einzige Wissenschaftstheoretiker, der die harten Wissenschaften als kulturell bedingt betrachtete und sie systematisch wie methodisch anderen Kulturen gleichstellte, war Ludwik Fleck. Zwar verwendet er nicht den Terminus $>$ Wissenskultur $<$, der Sache nach aber ist seine in den 1930er Jahren entstandene Denkstiltheorie der radikalste Versuch, ein Beschreibungsmodell für pluralistische Wissenskulturen $\mathrm{zu}$ entwickeln, in denen naturwissenschaftliches Wissen nicht mehr vor anderen Wissensformen eine 
Sonderstellung eingeräumt bekommt: »In der Naturwissenschaft gibt es gleichwie in der Kunst und im Leben keine andere Naturtreue als die Kulturtreue $\ll .{ }^{17}$ Man könne daher über die Wissenschaft $\gg$ nur so sprechen, wie wir das Wort >die Kunst< verwenden, um das Gemeinsame in den Bestrebungen von Musik, Malerei und Dichtung usw. zu belegen $\ll$. ${ }^{18}$

Ironischerweise wurde Flecks Theorie durch Thomas Kuhn wiederaufgenommen, dessen Paradigmentheorie indes sich nur auf Entwicklungen bezieht, die innerhalb der scientific communities der mathematischen Wissenschaften sich ereignen. Das Hineinspielen kultureller Vorgänge und Vorstellungen in die Produktion von naturwissenschaftlichen Wissen wird von Kuhn (im Unterschied zu Fleck) ebenso ausgeklammert, wie etwa die Rolle unbewusster Praktiken oder von Bildern. ${ }^{19}$ Doch Flecks Ideen überdauerten als Flaschenpost, sie wurden im breiten Strom der Kuhn-Rezeption an viele Küsten gespült und haben endlich in der vergangenen Dekade quer durch alle Disziplinen eine erstaunliche Renaissance erfahren.

Dieser erste Überblick eröffnet eine Chance, das Wissenskulturkonzept gegenüber all den Ansätzen zu profilieren, die gerade häufig zu seiner methodischen Grundlegung herangezogen werden: Gesucht wäre keine die harten Wissenschaften beiseite lassende Wissenssoziologie, keine auf Aussagensysteme fixierte Diskursanalyse, keine für die Naturwissenschaften reservierte historische Epistemologie oder Paradigmentheorie, sondern ein Konzept, dass die jeweiligen Beschränkungen der genannten Ansätze insofern überschreitet, als es einen Pluralismus sehr vieler Wissenskulturen akzeptiert und vordringlich solche dynamischen Formierungsprozesse untersucht, die sich nicht in fixen Strukturen, Ordnungen oder Diskursen des Wissens manifestieren, sondern in transitorischen Denkstilen, also in offeneren Gebilden, die von ästhetischen, nicht propositional fassbaren Faktoren mitkonstituiert werden.

Das Problem hierbei ist, dass man sich von der klassischen Wissensdefinition, die Wissen als wahre gerechtfertigte Meinung bestimmt, entfernt, zugleich aber am Wissensbegriffs fest- hält. Hier öffnet sich eine Kluft zwischen philosophischer Wissenstheorie und historischen Wissenskonzepten. Aus der Sicht der Philosophie muss eine Trennlinie zwischen Wissen und Meinung gezogen werden, Meinungen in einem Raum der Gründe gerechtfertigt, verschiedene Grade und Modi des Wissens differenziert werden. Der Begriff der Wissenskultur suggeriert zudem, dass auch wenn es viele verschiedene Wissenskulturen gibt, diese zumindest alle an einem gemeinsamen Wissen oder einer basalen universalen Rationalität teilhaben ${ }^{20}$ und verschleiert so die Möglichkeit, dass die Wissenskriterien der Objektivierbarkeit oder Intersubjektivierbarkeit nur innerhalb einer Wissenskultur gelten und zwischen den Kulturen unüberbrückbare Abgründe klaffen können. Auch ist es kein Einwand, dass Wissen offenbar durch verschiedene Wissenskulturen hindurch tradiert werden kann, denn die Übersetzung von Wissenselementen von einer Wissenskultur in einer andere muss ja nicht zwangsläufig unter Verweis auf allgemeine Gesetze oder eine reale Welt erfolgen, sondern schlicht durch Übernahme etablierter Praktiken in andere Kontexte. Allgemeinheit kommt also (eher) durch Stabilisierung und nicht (oder weniger) durch Rationalität zustande.

Mein Vorschlag wäre, den Ausdruck Wissenskultur nur dann zu verwenden, wenn man anzeigen will, dass es nicht ausschließlich epistemische Verfahren sind, die zur Auszeichnung von Meinungen als Wissen führen, sondern auch Faktoren, die außerhalb oder unterhalb des Raumes des Gebens und Nehmens von Gründen wirken, als kulturelle Praktiken, die Meinungen oder Glaubenssätze oder FürWahr-Haltungen generieren, festzurren und tradieren. Wissen ist nicht nur gerechtfertigte Meinung oder gedeutete Information, ${ }^{21}$ sondern ebenso unreflektiert übernommene Einstellung, fixiertes Vorurteil, Produkt von Dressur und Anpassung, Rhetorik und Strategie.

\section{Wissenskulturen, Kulturelles Wissen und Wissen}

Hans Jörg Sandkühler hat vor Kurzem zu Recht festgestellt, dass der Begriff der Wissens- 
kultur »durch den inflationären Gebrauch des Wortes >Kultur $<$ belastet $\ll$ ist. ${ }^{22}$ Mittlerweile könnte man sogar weiter gehen und sagen, durch den inflationären Gebrauch des Wortes $>$ Wissenskultur< sei auch der Wissensbegriff so belastet worden, dass man inzwischen die kulturelle Relativität des Wissens ${ }^{23}$ immer auch dann mitdenkt, wenn nur von Wissen die Rede ist. Doch was wäre > kulturelles Wissen<?

Im Anschluß an die die klassische Wissenssoziologie und an das Standardwerk von Berger und Luckmann ${ }^{24}$ hat bereits 1989 Titzmann $^{25}$ einen weiten Wissensbegriffs $\mathrm{zu}$ formulieren versucht, der neueren Entwicklungen in der Epistemologie Rechnung tragen kann. Als Minimaldefinition führt er ein, dass der Ausdruck $>$ kulturelles Wissen< kein $\gg$ Wissen von Individuen, sondern von Gruppen $\ll{ }^{26}$ bezeichnen solle und ergänzt:

$>$ Kulturelles Wissen< sei nun die Gesamtmenge der Propositionen, die die Mitglieder der Kultur für wahr halten bzw. die eine hinreichende Anzahl von Texten der Kultur als wahr setzt [...] Zum Wissen gehören also auch kulturelle Annahmen, von denen wir zu wissen glauben, daß sie falsch sind. ${ }^{27}$

Häufig sei es jedoch schwer zu unterscheiden, ob bestimmte Propositionen im epistemischen Modus des Glaubens oder Wissens auftreten, man solle jedoch versuchen, genau dies jeweils historisch zu bestimmen sowie differenzieren, ob ein allgemein geteiltes oder gruppenspezifisches Wissen vorliege. Zudem gebe es auch nicht-bewußte Anteile am Wissen, z.B. in Texten nicht explizit formulierte, aber implizit vorhandene Propositionen; diese sollen »als kulturell zumindest grundsätzlich als bewusstseinsfähig « gelten, also explizierbar sein. ${ }^{28}$ Titzmanns Vorschlag hat den Vorzug, dass er nicht jede Meinung zum Wissen erhebt, sondern die jeweiligen historischen Varianten, wie Wissen als Wissen ausgezeichnet wurde, gerade zum Untersuchungsgegenstand erklärt. Auch gilt ihm naturwissenschaftliches Wissen nicht als > natürliche Art $<{ }^{29}$ sondern auch dieses wird als abhängig von kulturellen Diskursen gedacht.
Von hier aus weitergedacht, wäre Wissen zu bestimmen als sozial und somit kontextuell gerechtfertigte Meinung $\mathrm{zu}$ betrachten. Die Wahrheitsbedingung fiele weg; es reiche, dass eine Gruppe bestimmte Propositionen für wahr halte. ${ }^{30}$ Doch auch die Rechtfertigungsbedingung gerät ins Wanken, wenn man - mit Fleck - davon ausgeht, dass Wissenskulturen nicht allein durch prinzipiell explizierbare Propositionen gefasst werden, sondern viele unwägbare und nur innerhalb eines Denkstils ${ }^{31}$ wirksame Faktoren wie soziale Stimmungen mit ihm Spiel sind und somit auch die Zusammenhänge zwischen einzelnen Elementen-und Motiven in einer Wissenskultur nicht kausal, sondern ästhetisch oder ethisch sich bilden und so für andere Gruppen kaum oder gar nicht nachzuvollziehen sind. ${ }^{32}$ Wie also soll man methodisch damit umgehen?

\section{Wissenskulturen, Wissensgeschichte und historische Epistemologie}

Während einer Auseinandersetzung mit Imre Lakatos gab Thomas Kuhn zu Bedenken,

daß wenn Geschichtswissenschaft oder eine andere empirische Disziplin uns $\mathrm{zu}$ der Überzeugung führt, daß die Entwicklung der Wissenschaft wesentlich von dem Verhalten abhängt, das wir früher für irrational gehalten haben, wir dann daraus schließen sollten, dass [...] unser Begriff von Rationalität hier und dort korrigiert werden muß. ${ }^{33}$

Nicht allzu viele Wissenschaftstheoretiker würden diesen Satz unterschreiben, und auch Kuhn ging bei dessen Selbstanwendung nicht sehr weit, denn aus ihm folgt, dass die Beschreibung historischer Wissensformen auf die eigene Wissenskonzeption rückwirkt und zur normativen Forderung führt, das Verhältnis von Geschichtsschreibung und Erkenntnistheorie stets $\mathrm{zu}$ reflektieren und gegebenenfalls letztere zu revidieren.

Nun sind in den vergangenen Jahrzehnten unter dem Titel einer Geschichte des Wissens und der Wissenskulturen eine Vielzahl histori- 
scher Wissen- und Wissenschaftskonzeptionen beschrieben worden, in denen irrationale Faktoren im Kuhnschen Sinne beteiligt sind, und dies hat in den kulturwissenschaftlichen Disziplinen zu einer Entgrenzung, ja Verwilderung des Wissensbegriffs geführt. Wir kennen nun epistemische Bilder und Metaphern, tacit knowledge, Poetologien des Wissens, unbewusstes Wissen, Körperwissen, Künstler- und Handwerkerwissen, epistemische Gefühle, denkende Augen und Hände, uvm.

Untersucht man Wissenskulturen historisch, betreibt man zwar meist keine Wissenschaftsgeschichte im engeren Sinne, aber immerhin doch Wissensgeschichte, d.h. ein Abglanz der wissenschaftlichen Wirklichkeitstreue und Allgemeingültigkeit soll auch auf das eigene Unternehmen fallen. Der Vielfalt der dadurch in Umlauf gekommenen Wissensbegriffe entspricht eine Vielzahl an historiographischen Modellen und Methoden, die in den diversen Disziplinen in Anschlag gebracht werden. Sie spiegeln zunächst lediglich wider, dass die Geschichte des Wissens nicht zielgerichtet ablief, sondern vielmehr sich als eine chaotische Menge einander überkreuzender Fährten präsentiert, die in verschiedene Richtungen weisen. Doch wenn wir sagen, all das sei irgendwie Wissen, können wir mit Wissensbegriff nicht mehr historisch arbeiten, seine Kontur verliert jede Schärfe und Funktionalität.

Mehr noch: Gerade im Bestreben, vergangene Weltanschauungen, mentale Zustände, künstlerische oder handwerkliche Fertigkeiten und deren Repräsentationsweisen als Wissensformen aufzuwerten, tappt man leicht in die Anachronismusfalle, wenn z.B. just das retrospektiv zum Wissen nobilitierte Können in der $\mathrm{zu}$ untersuchenden Wissenskultur gar nicht oder nur unter bestimmten Voraussetzungen als Wissen akzeptiert wurde.

Z.B. zeigt sich im Lichte der oben gebrachten Zitate aus Nietzsches Geburt der Tragödie, dass es dem Sprachgebrauch der Zeit widersprechend und daher sinnentstellend wäre, würde man die Entstehung dieser Schrift retrospektiv in einer Wissenskultur der Altphilologen im 19. Jahrhundert verorten wollen, denn man erkennt so nicht mehr, welche Begriffe von Kultur und Wissen seinerzeit zirkulierten. Oder schauen wir z.B. in den Codex Leicester, sehen wir, wie Leonardo immer wieder die Vorläufigkeit seiner Beobachtungen und das Tentative seiner Anordnung und Darstellung betont. Erst im Codex Atlanticus ${ }^{34}$ fallen Ausdrücke wie conoscere und sapere, und hier ist jeweils darauf zu achten, ob erst durch das Integrieren einer Beobachtung in einen theoretischen Rahmen, der Annahmen über NichtBeobachtbares enthält, diese Beobachtung als Wissen deklariert wird, oder ob bereits die Beobachtung und der sie zu fassende zeichnerische Entwurf als Wissen gelten können. In einer pauschalen Wissenszuschreibung - etwa durch die leichtfertige Rede von einer Artisanal Epistemology ${ }^{35}$ - verschwimmen gerade diese Differenzen.

Man muß den Dschungel der Wissensbegriffe ja nicht roden, doch um sich in ihm zu orientieren und gerade um seine Vielfalt $\mathrm{zu}$ erkennen, und vor allem, um in den Blick zu bekommen, wie historisch auf unterschiedliche Weisen Wissen von Nicht-Wissen oder bloßen Fürwahrhalten geschieden wurde, oder ob erhobene Wissensansprüche nach damaligen Kriterien auch eingelöst werden konnten, müssen wir die Wissensbegriffe trennscharf halten.

Hierzu bieten sich zwei Hauptvarianten an: Wir können versuchen eine Typologie unterschiedlicher Wissenskonzeptionen $\mathrm{zu}$ entwerfen, wobei wir dann doch immer noch angeben können müssten, wodurch hier auf je unterschiedliche Weise das Wissen zum Wissen wird und wieso diese Rekonstruktion nun eine Wissensgeschichte ist und nicht schlicht GeistesIdeen-, Mentalitäts-, Diskurs-, Technik- oder Bildungsgeschichte, oder Ikonologie etc. Bei näherem Zusehen wird man feststellen, dass viele der heute als Wissensgeschichte auftretenden Historiographien, zumal wenn sie weitgehend disziplinimmanent und an einem einheitlichen Gegenstand orientiert bleiben, ohne weiteres unter einem der alten Namen geführt werden könnten. Als Lackmustest für die historische Betrachtung von Wissensformen könnte man vielleicht ein Gedankenexperiment vorschlagen: Ist es möglich z.B. statt $>$ Wissen im 
Entwurf $<$ auch $>$ Meinung im Entwurf $<$ sagen, oder von Handwerkerkulturen statt von $>$ Wissenskulturen der Handwerker< sprechen, ohne dass sich etwas wesentliches änderte?

Der zweite Weg ist: Man kann versuchen, den klassischen Wissensbegriff zu erweitern, bis er für die historische Arbeit praktikabel wird. Nun droht jedoch, dass mit jedem Erweiterungsschritt gleich eine ganze Schule von Philosophen von der Fahne geht, die mit ihm gerade das Wissenskriterium preisgegeben sehen, sowie dass der Wissensbegriff zu weit wird und seine Anwendbarkeit einbüßt.

So lesen wir in einem aktuellen Aufsatz eines renommierten Historikers zum Thema: Was kann die Geschichtswissenschaft vom Wissen wissen?

Unter Wissen verstehe ich die Gewißheit, daß Phänomene wirklich sind und bestimmbare Eigenschaften haben. [Dies gelte für] alle Arten von >Wissen<: für das Alltags- und Jedermannswissen ebenso wie für Sonderwissen aller Art und für Expertenwissen [...]: für wahres und falsches Wissen, für Glaubenswisssen und wissenschaftliches Wissen. ${ }^{36}$

Wie repräsentativ diese Definition ist, mag jeder selbst beurteilen, doch allein dass sie möglich ist, sagt einiges aus: Wird Wissen auf eine Gewissheitsempfindung gegründet und unterstellt, diese korreliere mit einer Wirklichkeit da draußen, hat man alle Kriterien aus der Hand geschlagen. Nun kann wirklich alles zum Wissen werden. Aber wie können wir wissen, ob wir etwas wissen? Und was ist Wirklichkeit? Von wo aus gesehen gibt es falsches Wissen? Vielleicht kommen wir weiter, wenn wir versuchen, allerdings mit Siebenmeilenstiefeln, einen Schritt nach dem andern zu machen: In Platons klassischer Definition von Wissen war dieses bekanntlich nicht nur der Wahrnehmung (aisthesis) und Meinung (doxa) gegenübergestellt, sondern sogar der wabren Meinung. Diese muß erst noch gerechtfertigt werden. Es ist klar, dass dieser Wissensbegriff auf ein Ideal des perfekten Wissens zielt und von den kon- kreten epistemischen Praktiken des Erzeugens, Erwerbens, Prüfens, Ordnens, Darstellens, Etablierens und Tradierens von Wissen ${ }^{37}$ absieht. Letztere könnte man als wissenskulturelle Aspekt des Wissens bezeichnen. Doch auch jede Untersuchung der Frage, was das perfekte Wissen ist, wurde in bestimmten Kontexten durchgeführt, wodurch sich im Lichte der wissenskulturellen Perspektive die Wahrheit relativiert.

Streicht man die Wahrheitsbedingung, bleibt die Rechtfertigungsbedingung. Hier gab es ungezählte Versuche, diese systematisch oder historisch sozial und kulturell zu kontextualisieren, etwa indem man Wissen in einem Raum des Gebens und Nehmens von Gründen situierte. ${ }^{38}$ Bei dieser Konzeption liegt es auf der Hand, dass man auf basale Rationalitätsbedingungen des Verstehens rekurrieren muß, da argumentative Begründungsverfahren den Ausschlag für die Auszeichnung von Wissen geben. Aber auch der beschworene Raum des Gebens und Nehmens von Gründen hat mit der Normalwissenschaft wenig zu tun, sondern beschreibt die Wissenschaft in ihrer papiernen Gestalt. Wissen setzt sich häufig nicht durch bessere Argumente durch, Wissenskulturen bilden sich auch aufgrund von nichtpropositional explizierbaren Stimmungen, Begriffsfärbungen, kulturellen Wahrnehmungsdispositonen, Moden. Also wäre auch die Rechtfertigungsbedingung aufzugeben; es bleibt: Wissen als fixierte Meinung. ${ }^{39}$ Wissenskulturen konstituierten sich dann aus Prozeduren der kollektiven Fixierung von Fürwahrhaltungen. Beim Fixieren der Meinung sind offenkundig nicht-propositionale Faktoren beteiligt. Gesucht wäre also eine methodisches Verfahren, das es erlaubt, jene historischen und kulturellen Vorgänge transparent zu machen, durch die sich solche Inseln zeitweiliger Stabilität im Meere der Verwirrung bilden.

Dies zur Aufgabe macht sich die Historische Epistemologie. Im Hinblick auf deren Verhältnis zu den real existierenden Wissensgeschichtsschreibungen sind jedoch einige Klarstellungen nötig, da man sie oft vorschnell in Opposition zu diesen bringt und für die Rekonstruktion von Wissenskulturen in Dienst 
nimmt: Epistemologie bezeichnet traditionellerweise eine Erkenntnistheorie, die prüft, wodurch Wissen zum wissenschaftlichen Wissen wird. ${ }^{40}$ Auch in der historischen Epistemologie französischer Provenienz, namentlich bei Bachelard, fungiert die Epistemologie selegierend:

Der Epistemologe muß [...] die Dokumente, die der [Wissenschafts]Historiker gesammelt hat, auslesen. Er muß sie aus der Sicht der Vernunft beurteilen, ja aus der Sicht der entwickelten Vernunft, denn allein von unserer Zeit her können wir die Irrtümer der Vergangenheit des Geistes richtig beurteilen [...] Der Wissenschaftshistoriker muß die Ideen als Tatsachen nehmen. Der Epistemologe muß die Tatsachen als Ideen nehmen, indem er sie in ein Denksystem einfügt. Eine Tatsache, die von einer Epoche falsch interpretiert wurde, bleibt für den Historiker eine Tatsache, in der Sicht des Epistemologen ist sie ein Hindernis, ein Konter-Gedanke. ${ }^{41}$

Bachelard sieht die Aufgabe des Epistemologen also vordringlich darin, in der Geschichte das >Streben nach Rationalität und Konstruktion $\ll{ }^{42}$ herauszupräparieren. Verblüffend blind zeigt er sich gegenüber den ästhetischen Faktoren, die in die wissenschaftliche Praxis einwirken:

Eine Wissenschaft, die die Bilder hinnimmt, wird mehr als jede andere Opfer von Metaphern. Auch der wissenschaftliche Geist muß unablässig gegen die Bilder, die Analogien, die Metaphern ankämpfen. ${ }^{43}$

In der Gestalt, die die Historische Epistemologie bei Bachelard angenommen hatte, steht diese heutigen Ansätzen, Wissenskulturen zu beschreiben, genauso fern wie die die traditionelle Wissenschaftsgeschichte, der häufig ein engerer Wissensbegriff zugeschrieben wird. Nun ist es bereits schwierig, ein Kriterium zu finden, wie man wissenschaftliches Wissen von anderen Wissensformen abgrenzen kann, zumal solche Unterscheidungen vergleichsweise jung sind und daher von den historischen Zeugnissen nicht gestützt werden. Das kulturwissenschaftliche Mantra vom Einbezug nicht-propositionaler Formen des Wissens, die das propositionale Wissen ergänzen, korrigieren, oder mit ihm als alternative Wissensform konkurrieren können sollen, mündet selten in Studien, die konkret deren kognitiven Mehrwert aufzeigen. Zudem setzt es voraus, es gebe eine klare Vorstellung dessen was das propositionale Wissen, zu dem man andere Wissensarten in Beziehung setzt, sei, und unterstellt meist, dieses sei exemplarisch in den Wissenschaften anzutreffen. In negativer $\mathrm{Ab}$ grenzung bestätigt man ein naives Wissenschaftsideal, wobei unter anderem erstens die Einheit der Wissenschaft vorausgesetzt wird, zweitens dass im Unterschied zu Metaphern oder Bildern die wissenschaftlichen Begriffe eindeutig seien, drittens dass die Wissenschaften primär als Satzsysteme zu betrachten wären, die viertens, wenn durch sie Wissen einmal präzise gefasst und bewiesen ist, universelle und zeitlose Gültigkeit besäßen. Wenn sich jedoch zeigte, was Kuhn befürchtet hatte, nämlich dass auch für die wissenschaftliche Wissensproduktion >irrationale< Faktoren konstitutiv sind, stellt sich die Lage anders dar. Wissenschaftsgeschichte und allgemeinere Wissensgeschichte unterminieren beide die epistemologischen Wissensideale, und wissenschaftliches Wissen wäre ebenfalls nur mit einem weiten Wissensbegriff zu fassen.

\section{Eine Theorie der Wissenskultur}

Exemplarisch für dieses Vorgehen kann die Wissenschaftstheorie Ludwik Flecks gelten, die man insofern als Historische Epistemologie bezeichnen kann, als sie historisch aufzeigen will, unter welchen kulturellen und sozialen Bedingungen Wissen zu Wissen wird, aber dabei ohne den Fortschrittsglauben Bachelards auskommt.

Flecks Idee war, nicht nur die konkreten äußeren Umstände der Wissensproduktion zu untersuchen, sondern auch die Rolle von un- 
bewußt übernommenen Einstellungen aus Tradition und sozialer Umgebung mit einzubeziehen, um so zu zeigen, dass selbst die rein sachlich begründet erscheinenden Problemstellungen, Überprüfungs- und Rechtfertigungsverfahren der Wissenschaften kulturell geprägt und insofern prinzipiell stilbedingt sind. Davon nicht ausgenommen seien insbesondere auch Rekurse auf vermeintlich objektive wissenschaftliche Tatsachen. Flecks radikale Pointe dabei ist: Nicht nur darüber, was als eine Tatsache gilt, sondern auch darüber, was eine Tatsache ist, entscheidet der jeweilige Denkstil lokaler Denkkollektive.

In seinen Augen gibt es folglich nicht die Wissenschaft auf der einen und den soziale Kontext auf der anderen, somit auch keine nachzuweisenden oder zu bestreitenden Kausalrelationen dazwischen, schon gar keine unabhängigen Tatsachen, sondern lokale Dynamiken und Praktiken des Wissens, die sich über die Zirkulationen von Ideen und Tatsachen, die soziale Schulung des Sehens und kollektives Handeln konstituieren. Wissenschaft ist kulturelle Praxis; ${ }^{44}$ die kulturelle Praxis ist stilbedingt, verschiedene Denkstile charakterisieren unterschiedliche wissenschaftliche Kollektive, nie den Denkstil eines einzelnen; insofern könnte man Flecks Denkstilbegriff als ein Synomym für den Begriff der Wissenskultur begreifen. Dann gäbe es so viele Wissenskulturen wie Kollektive, die durch einen gemeinsamen Denkstil regiert werden, und zwar gleich $o b$ in der Wissenschaft oder anderswo.

Doch gerade weil Flecks Beispiele dennoch primär aus der Wissenschaftsgeschichte stammen, gilt, dass wenn mit Hilfe seines Beschreibungsmodells erkennbar wird, dass die Genese und die Geltung wissenschaftlichen Wissens an spezifische, räumlich und zeitlich lokale Umstände geknüpft ist, und dabei von einer Vielfalt anderer wissenschaftlicher, kultureller und sozialer Handlungsfelder abhängt, dieses Modell dann auch auf alle andere Spielarten des Wissens übertragen werden kann.

Flecks Paradebeispiel ist die Syphilis, - diese empfand man von Beginn an als entehrende Krankheit, die mit dem Gepräge der Sünde be- lastet war und die das Blut vergiftete. Eine solche spezifisch emotional geprägte Vorstellung nennt Fleck eine >Präidee<. Solche Präideen sind keine Archetypen, sondern nur allgemeine vage Grundvorstellungen, die der Entwicklung eines Forschungsfeldes eine ungefähre Richtung vorgeben. Die ursprüngliche Idee kann sich komplett wandeln, aber dennoch weiter Einfluss behalten, etwa indem sie als mythische Vorstellung im Kollektiv der Syphilisforscher herumspukt und deren Forschung in die Richtung lenkt, gerade im Blut nach Erregern zu suchen und sie dann glauben macht, es sei Ihnen tatsächlich der Nachweis gelungen. Hinzu kommt, dass weil die Syphilis als Lustseuche in der europäischen Kultur eine besondere ethische Färbung hat, entsprechend die Forschung sich stärker auf sie als auf andere nicht minder schlimme Seuchen konzentriert.

Unter anderem auch dieses - ansonsten stets ausgeblendete - Einwirken von in der jeweiligen lokalen Kultur zirkulierenden Bilder und Vorstellungen, die etwa präsent waren durch in den Städten angeschlagene Plakate, die vor der Syphilis warnten, Filme und Theaterstücke, die über die Syphilis ${ }^{45}$ aufklärten, hätte eine Beschreibung der Wissenskultur der Serologen miteinzufangen. Hierbei kommt es nicht auf einzelne Wissenschaftler an, sondern auf ihre soziale Gemeinschaft, denn die Quelle des Denkens eines Forschers

liegt gar nicht in ihm, sondern in der sozialen Umwelt, in der er lebt, in der sozialen Atmosphäre, in der er atmet und er kann gar nicht anders denken als so, wie es aus den in seinem Hirn sich konzentrierenden Einflüssen der ihn umgebenden sozialen Umwelt mit Notwendigkeit sich ergibt. ${ }^{46}$

Fleck zufolge formieren sich solche Gemeinschaften durch die Ausbildung eines einheitlichen Denkstils, der sich auch darin manifestiert, dass aufgrund der kulturellen Prägungen nur die Mitglieder des eigenen Kollektivs in der Lage sind, spezifische Stilfärbungen herauszufühlen; es kommt zu einer »Stimmungskameradschaft $\ll{ }^{47}$ Die Stimmung erzeugt eine 
Bereitschaft zum gerichteten Wahrnehmen, Bewerten und Anwenden des Wahrgenommen, sie ist die Triebkraft denkstilgemäßen kollektiven Handelns.

Nach Fleck müssen daher weitere Analyseschritte folgen, denn es gilt auch zu zeigen, wie von solchen Stimmungen die konkrete Forschungsspraxis geprägt wird, welche besonderen Metaphern und Begriffe aus ihr heraus gewählt werden, um die Blutproben zu beschreiben, wie bestimmte Gestalten aus den Proben herausgeschaut und in kollektiven Verhandlungen fixiert werden, wie dann dieses esoterische Wissen in Zeitschriftenaufsätzen verbreitet, in Lehrbüchern kodifiziert und in allgemeinen Darstellungen popularisiert wird, um schliesslich selbst zum kulturellen Faktor zu werden, der wiederum auf die Forscher zurückwirkt, die nun bereit sind, ihre ursprünglich vage formulierten Ideen als Tatsachen $\mathrm{zu}$ glauben.

Flecks Denkstiltheorie beschränkt sich folglich keineswegs auf das Denken, sondern schließt das Wahrnehmen sowie die Praktiken und Instrumente eines Kollektivs mit ein, und alle diese Faktoren sind bei der Wissensproduktion immer mit im Spiel. Sei ein Denkstil erst einmal etabliert, werde er tradiert und dies nicht durch bessere Argumente, sondern durch Dressur. Daher lassen sich Fleck zufolge die wissenskulturellen Faktoren, das Unbestimmte, das Unbewusste, die sozialen Zwänge, die das Entdecken, Prüfen, Rechtfertigen und Tradieren von Wissen determinieren, nicht durch Korrekturverfahren nachträglich herausfiltern, sondern werden als nicht-eliminierbare denkstiltransformierende Größen mit epistemologischer Funktion einsichtig und dadurch eben auch zum Gegenstand wissensgeschichtlicher Forschung erhoben.

Flecks Denkstile sind somit weder Methoden noch allgemeine Seh- oder Denkformen; sie bezeichnen keine Epochen, Diskursformationen oder Weltanschauungen und charakterisieren auch keine sozialen Gruppen, Institutionen oder wissenschaftlichen Einzelleistungen, auch keine statischen begrifflichen oder epistemischen Konstellationen, sondern jene Vor- gänge, die Wissen generieren und Wissenskulturen formieren.

Einige Wissenschaftsphilosophen werden die Einsicht nur ungern zur Kenntnis nehmen, dass sich Fleck zufolge die Entwicklung und Formierung der Wissenschaften nicht primär unter Bezug auf allgemeine Erkenntniskriterien und Methoden rekonstruieren läßt, sondern dass auch die jeweilige wissenschaftliche Tatsachenproduktion erst mit kultur- und literaturwissenschaftlichen Analysetechniken aufgeklärt werden kann. Ein weiterer Vorzug der Theorie Flecks ist, dass einzelne Denkstile in ihrer Entwicklung und ihrer Differenz zueinander betrachtet werden können. Dies führt in Flecks Augen nicht zu einem kognitiven Relativismus, sondern nur $\mathrm{zu}$ einem »internrealistischen epistemologischen Pluralismus $\ll .{ }^{48}$

>Die Wahrheit< als eine aktuelle Etappe der Denkstilumwandlng ist immer nur die eine: Sie ist durch den Stil restlos determiniert. Die Verschiedenheit der Wirklichkeitsbilder ist einfach eine Folge der Verschiedenheit der Erkenntnisobjekte in verschiedenen Denkstilen. ${ }^{49}$

Dies verändert die wissenshistorische Suchoptik: Intuitiv erschaute Analogien zwischen Denkstilen weist Fleck zurück: hier werde Ungleiches gleichgesetzt. Ebenso gibt es für Fleck keine allgemeinen Diskursregeln, die alle Bereiche einer Wissenskultur einer Epoche organisieren. Seine vergleichende Denkstilforschung zielt darauf $a b$, die Besonderheiten und verschiedenen Geschwindigkeiten vieler einzelner Wissenskulturen hervorzuheben sowie die Differenzen zu anderen Denkstilen bis hin zu Inkommensurabilität zu beschreiben und nicht darauf eine allgemeine Metatheorie für Denkstile oder Ordnungen der Dinge einer Wissenskultur aufzustellen. Eine vergleichende Denkstilforschung versucht hingegen, diejenigen Denkzwänge transparent zu machen, aufgrund derer die Mitglieder einer anderen Wissenskultur Tatsachen für evident halten, die einem selbst opak vorkommen und vice versa.

Das Vergleichen verschiedener Standpunk- 
te zielt nicht auf ein objektives oder synthetisches Gesamtbild, sondern führt zu einem Nebeneinander von widersprechenden, nicht zusammenstimmenden Bildern, durch jedoch die die historischen Varianten wie jeweils Wissen von Nichtwissen, von Meinungen, von Teilwissen etc. geschieden und einander zu oder abgesprochen wurde, rekonstruiert werden können. In Flecks Augen gibt es daher keine besseren oder schlechteren epistemischen Kontexte. Um diese Unterscheidung vornehmen zu können, müsste man eine basale universale Rationalität unterstellen, und diese gebe es nur qua Verabsolutierung des Wissensideals und der Basisintuitionen eines partikularen Denkstils. Für Fleck wäre dies Denkstilfanatismus. Die Übergänge und Wechselwirkungen im denkstilkonstituierenden Kreislauf von Wahrnehmung, gezielten Beobachtungen, deren Transposition in Bilder und Sprache, wären je nach Forschungsgebiet anders $\mathrm{zu}$ beschreiben, jedoch kaum je disziplinimmanent $\mathrm{zu}$ verfolgen oder gar einzufangen.

Flecks Denkstiltheorie versucht der Komplexität ihres Gegenstands insofern Rechnung zu tragen, als sie diese mit in ihre eigene Methodologie aufnimmt, und ihrerseits wissenschaftshistorische, philosophische, soziologische, kunsthistorische, kultur- und literaturwissenschaftliche, Perspektiven verbindet. Das Vorgehen ist weder Autor- noch werk- oder Institutionenzentriert, sondern es verfolgt u.a. Forschungsrituale, Zitatwege, intertextuelle und intermediale Verweisungen, um anhand ihrer Transformationen in Abschriften, Illustrationen, Zeichnungen, Editionen, Übersetzungen und Briefen die allmähliche Formierung einer Wissensform zu beschreiben. Die Denkstiltheorie erscheint daher als der bislang praktikabelste Vorschlag, um historisch wie systematisch Wissenskulturen im Hinblick auf die beiden sie konstituierenden Elemente $\mathrm{Kul}$ tur und Wissen zu begreifen.

\section{Anmerkungen}

${ }^{1}$ In den letzten Jahren eingerichtet wurden z.B. der Masterstudiengang $>$ Historische Wissenskul- turen < der Universität Rostock und der interdisziplinäre Masterstudiengang >Wissenskulturen< der TU Stuttgart.

2 http://phil-kult.univie.ac.at/forschung/kulturenund-identitaeten-im-aussereuropaeischen-raum/ fruehneuzeitliche-osmanische-wissenskultur/

${ }^{3}$ C. FEEST, Wampum, Wert und Wissen. Zur Wissenskultur der Irokesen, in: J. FRIED, TH. KAILER (Hrsg.), Wissenskulturen. Beiträge zu einem forschungsstrategischen Konzept, Akademie Verlag, Berlin 2003, S. 87-103.

${ }^{4}$ C. MAREIS, Design als Wissenskultur. Interferenzen zwischen Design- und Wissensdiskursen seit 1960, Transcript, Bielefeld 2011.

${ }^{5}$ G. BRANDSTETTER, Tanz als Wissenskultur. Körpergedächtnis und wissenstheoretische Herausforderung, in: S. GEHM, P. HuSEMANN, K. VON WilcKe (Hrsg.), Wissen in Bewegung. Perspektiven der künstlerischen und wissenschaftlichen Forschung im Tanz, Transcript, Bielefeld 2007, S. $37-$ 48; S. HusCHKA (Hrsg.), Wissenskultur Tanz: Historische und zeitgenössische Vermittlungsakte zwischen Praktiken und Diskursen, Transcript, Bielefeld 2009.

${ }^{6}$ I. MAREI KRÜGER-FÜRHOFF, Verpflanzungsgebiete. Wissenskulturen und Poetik der Transplantation, Wilhelm Fink Verlag, München 2012.

${ }^{7}$ H. PAHL, Die Kirche im Dorf. Religiöse Wissenskulturen im gesellschaftlichen Wandel des 19. Jahrbunderts, Akademie Verlag, Berlin 2006.

${ }^{8} \mathrm{Y}$. WÜBBEN, Kontraste und Synergien? Außeruniversitäre Wissensdistribution am Beispiel der Halleschen Lehrprosa (um 1750), in: J.-M. VALENTIN (Hrsg), Akten des XI. Internationalen Germanistenkongresses, Bd. V: Kulturwissenschaft versus Philologie? - Wissenschaftskulturen: Kontraste, Konflikte, Synergien - Editionsphilologie, in: «Jahrbuch für Internationale Germanistik», Reihe A, Bd. LXXXI, Peter Lang, Bern 2008, S. 239-248, hier S. 246.

${ }^{9}$ Vgl. K. KNORR Cetina, Epistemic Cultures: How the Sciences Make Knowledge, New York 1999.

${ }^{10}$ Vgl. J. FRIED, TH. KeILER (Hrsg.), Wissenskulturen. Beiträge $z u$ einem forschungsstrategischen Konzept, Akademie Verlag, Berlin 2003.

${ }^{11}$ W. DETEL, Wissenskulturen und universelle Rationalität, in: J. FRIED, M. STOLLEIS (Hrsg.), Wissenskulturen. Über die Erzeugung und Weitergabe von Wissen, Campus Verlag, Frankfurt a.M.-New York 2009, S. 181-214, hier S. 181.

${ }^{12}$ B. DOTZLER, Explorationen. Literaturforschung und die Geschichte des Wissens und der Wissen- 
schaften, in: Berlin-Brandenburgische Akademie der Wissenschaften. Berichte und Abhandlungen, Bd. IX, Akademie Verlag, Berlin 2002, S. 311-327. ${ }^{13}$ Ebd. S. 311.

${ }^{14}$ F. NIETZSCHE, Die Geburt der Tragödie aus dem Geiste der Musik (1872), in: F. NIETZSCHE, Kritische Studienausgabe in 15 Bände, Bd. I, hrsg. von C. Colli, M. MONTINARI, München 1980, S. 117 (Hervorhebung: CZ). Zur tragischen Kulturtheorie des jungen Nietzsche siehe: C. ZITTEL, Selbstaufhebungsfiguren bei Nietzsche, Königshausen \& Neumann, Würzburg 1995.

${ }^{15} \mathrm{M}$. SCHELER, Wissensformen und die Gesellschaft, Der neue Geist, Leipzig 1926, S. 186.

${ }^{16}$ Vgl. dazu C. ZITTEL, Von der Ordnung der Diskurse und ibrer Subversion durch die Bilder. Blinde Flecken in Foucaults Diskursanalyse, in: F. EDER, A. LANDWEHR, P. SARASIN (Hrsg.), Bilder in historischen Diskursen, in press.

${ }^{17}$ L. FLECK, Die Entstehung und Geschichte einer wissenschaftlichen Tatsache (1935), hrsg. von L. SCHÄFER, TH. SCHNELLE, Suhrkamp, Frankfurt a.M. 1980, S. 48.

${ }^{18}$ L. FLECK, Wissenschaftstheoretische Probleme (1946), in: L. FlECK, Denkstile und Tatsachen, hrsg. von S. WERnER, C. ZITTEL, Suhrkamp, Berlin 2011, S. 369-389, hier S. 369. Wenn also Mareis (MAREIS, Design als Wissenskultur. Interferenzen zwischen Design- und Wissensdiskursen seit 1960, a.a.O., S. 217) beispielsweise >Design< als dritte Wissenskultur neben den Kulturen der Natur- und Geisteswissenschaften etabliert sehen will und sich dabei auf Fleck beruft (ebd., S. 217f.), verkennt sie dessen basale Einsicht, dass wenn Wissenschaften als kulturelle Praktiken aufgefasst werden, die Unterscheidung zwischen Natur- und Geisteswissenschaften hinfällig ist und stattdessen von der Koexistenz sehr vieler verschiedener kleinerer und größerer Wissenskulturen ausgegangen werden muss.

${ }^{19}$ TH. KuHn, Comment on the Relations of Science and Art, in: TH. KUHN, The Essential Tension: Selected Studies in Scientific Tradition and Change, Chicago 1977, S. 340-351 (dt. Bemerkungen zum Verhältnis von Naturwissenschaft und Kunst, in: TH. KUHN, Die Entstehung des Neuen, hrsg. von L. KRÜGER, H. VETTER, Suhrkamp, Frankfurt a.M. 1978, S. 446-460, hier S. 448).

${ }^{20}$ Vgl. W. DETEL, Wissenskulturen und universelle Rationalität, in: J. FRIED, M. STOLLEIS (Hrsg.), Wissenskulturen. Über die Erzeugung und Weitergabe von Wissen, a.a.O., S. 181-214.
${ }^{21}$ Inkonsequent ist, wenn man einerseits Wissenskulturen beschreibt, indem man auf die Tradierens, Verdichtens, Objektivierens, Deutens von und Reflektieren auf Wissen verweist, um jene Prozesse zu bezeichnen, die Wissen hervorbringen sollen, andererseits die Wissenskultur primär über Wissensordnungen zu definieren und dabei den Wissensbegriff in einer klassischen Version zu retten. Breidbach z.B. nennt sowohl die impliziten, unbewussten Faktoren, die bei der Bildung von Wissenskulturen hineinspielen, gleichwohl liefert er Definitionen im Indikativ, wie: »Wissen ist interpretierte Information $\ll$, die mit neuen problematischen Termini aufwarten und kaschieren, dass jenseits der Deutungen und Begründungen auch andere Faktoren wie Dressur und Stimmungen dafür sorgen, dass manche Meinungen für wahres Wissen gehalten werden. Vgl. O. BREIDBACH, Neue Wissensordnungen, Suhrkamp, Frankfurt a.M. 2008, S. 14.

${ }^{22}$ H.J. SANDKÜHLER, Kritik der Repräsentation. Einfübrung in die Theorie der Überzeugungen, der Wissenskulturen und des Wissens, Suhrkamp, Frankfurt a.M. 2009, S. 69.

${ }^{23}$ Ebd., S. 70 und $165 f$.

${ }^{24}$ P. Berger, Th. LuCkmann, The Social Construction of Reality, Garden City, New York 1966 (dt. Die gesellschaftliche Konstruktion der Wirklichkeit. Eine Theorie der Wissenssoziologie, hrsg. von H. PLESSNER, M. PLESSNER, Fischer, Frankfurt a.Main 1987).

${ }^{25}$ M. TitzmanN, Kulturelles Wissen - Diskurs Denksystem: Zu einigen Grundbegriffen der Literaturgeschichtsschreibung, in: «Zeitschrift für französische Sprache und Literatur», Jg. XCIX, H. 1, 1989, S. 47-61.

${ }^{26}$ Ebd., S. 47.

${ }^{27}$ Ebd., S. 48.

${ }^{28}$ Ebd., S. 49.

${ }^{29}$ Siehe dazu R. RoRTY, Is Natural Science a Natural Kind?, in: E. MCMULLIN (ed.), Construction and Constraint. The Shaping of Scientific Rationality, Notre Dame University Press, Notre Dame (ILL) 1988, pp. 49-74 (dt. Ist Naturwissenschaft eine natürliche Art?, in: R. RORTY, Eine Kultur ohne Zentrum, hrsg. von J. SCHULTE, Reclam, Stuttgart 1993, S. 13-47).

${ }^{30}$ Vgl. A. BECKER, Kann man Wissen konstruieren?, in: C. ZITTEL (Hrsg.), Wissen und soziale Konstruktion, Akademie Verlag, Berlin 2002, S. 15-25, hier S. 16; C. ZITTEL, Konstruktionsprobleme des Sozialkonstruktivismus, in: C. ZITTEL (Hrsg.), Wis- 
sen und soziale Konstruktion, a.a.O., S. 87-108.

${ }^{31} \mathrm{Zu}$ den Implikationen des Begriffs >Denkstil< siehe: C. ZITTEL, Ludwik Fleck und der Stilbegriff in den Naturwissenschaften. Stil als wissenschaftshistorische, epistemologische und ästhetische Kategorie, in: H. BREDEKAMP, J. KROIS (Hrsg.), Sehen und Handeln, Akademie Verlag, Berlin 2011, S. 171-206.

${ }^{32}$ R. MusIL, Das Hilflose Europa oder Reise vom Hundertsten ins Tausendste (1922), in: R. MUSIL, Gesammelte Werke, Bd. VIII, hrsg. von A. FRISÉ, Rowohlt, Hamburg 1989, S. 1075-1094.

${ }^{33}$ TH. KUHN, Notes on Lakatos, in: «Boston Studies in the Philosophy of Science», vol. VIII, 1971, pp. 137146 (dt. Anmerkungen zu Lakatos, in: W. DIEDERICH (Hrsg.), Theorien der Wissenschaftsgeschichte, Suhrkamp, Frankfurt a.M. 1974, S. 120-133.

${ }^{34}$ Vgl. L. DA VINCI, Codex Atlanticus: Studien, Skizzen und Zeichnungen, hrsg. von M. NAVONI, Bucher, München 2012.

${ }^{35}$ P. SMITH, Art, Science, and Visual Culture in Early Modern Europe, in: «Isis», Jg. XCVII, H. 1, 2006, S. 83-100; P. SMITH, Artisanal Epistemology, in: P. SMITH, The Body of the Artisan, Art and Experience in the Scientific Revolution, Chicago University Press, Chicago 2006, S. 59-93; P. SMITH, Artists as Scientists: Nature and Realism in Early Modern Europe, in: «Endeavour», vol. XXIV, n. 1, 2000, S. 13-21.

${ }^{36}$ G. OEXLE, Was kann die Geschichtswissenschaft vom Wissen wissen, in: A. LANDWEHR (Hrsg.), Geschichte[n] der Wirklichkeit. Beiträge zur Sozialund Kulturgeschichte des Wissens, Wißner Verlag, Augsburg 2002, S. 31-60. Siehe auch die catch allDefinition in R. KLAUSNITZER, Literatur und Wissen: Zugänge - Modelle - Analysen, W. De Gruyter, Berlin 2008: Wissen läßt sich »als Gesamtheit von begründeten (bzw. begründbaren) Kenntnissen begreifen, die innerhalb kultureller Systeme durch Beobachtung und Mitteilung, also durch Erfahrungen und Lernprozesse erworben sowie weitergegeben werden und einen reproduzierbaren Bestand von Denk-, Orientierungs- und Handlungsmöglichkeiten bereitstellen [...] Wissen ist die dynamische Gesamtheit aller Vorgänge und Resultate, in denen sich regelgeleitete Umgangsweisen mit begründeten Erkenntnissen auf Grundlage symbolischer Ordnungen und Technologien formieren und vollziehen, in Wirkung treten und verändern « (ebd., S. 12f.).

${ }^{37}$ Siehe dazu W. DETEL, Wissenskulturen und episte- mische Praktiken, in: J. FRIED, TH. KEILER (Hrsg.), Wissenskulturen. Beiträge zu einem forschungsstrategischen Konzept, Akademie Verlag, Berlin 2003, S. 119132; W. DETEL, Der Sozialkonstruktivismus und die Wissenschaftsgeschichtsschreibung des 17. Jahrhunderts, in: C. ZITTEL (Hrsg.), Wissen und soziale Konstruktion, a.a.O., S. 67-86.

${ }^{38}$ Vgl. z.B. W. SELLARS, Empiricism and the Philosophy of Mind (1956), Cambridge (MA) 1997; R. BRANDOM, Making It Explicit. London/Cambridge (MA) 1994; K. DeRose, Contextualism and Knowledge Attributions, in: «Philosophy and Phenomenological Research», Jg. LII, H. 4, 1992, S. 913-929; M. WILLIAMS, Unnatural Doubts: Epistemological Realism and the Basis of Scepticism, Princeton University Press, Oxford 1991.

${ }^{39} \mathrm{Zu}$ diesem Problemkomplex siehe: J. ROSENBERG, Was epistemische Externalisten vergessen, in: C.U. Moulines, K.-G. Niebergall (Hrsg.) Argument und Analyse, Mentis Verlag, Paderborn 2002, S. 15-31; J. ROSENBERG, Thinking about Knowing, Oxford University Press, Oxford 2002, besonders Kap. VII.

${ }^{40}$ H.J. RHEINBERGER, Einführung in die historische Epistemologie, Junius Verlag, Hamburg 2007, S. 11.

${ }^{41}$ G. BACHELARD, La formation de l'esprit scientifique, Vrin, Paris 1938 (dt. Die Bildung des wissenschaftlichen Geistes, hrsg. von M. BISCHOFF, W. LEPENIES, Suhrkamp, Frankfurt a.M. 1987, S. 51). ${ }^{42}$ Ebd.

${ }^{43}$ Ebd., S. 80.

${ }_{44}$ Vgl. dazu z.B. die Beiträge in M. Epple, C. ZITTEL (Hrsg.), Science as Cultural Practice, vol. I, Cultures and Politics of Research from the Early Modern Period to the Age of Extremes, Akademie Verlag, Berlin 2010.

${ }^{45}$ Vgl. dazu J. LAZARDZIG, Inszenierung wissenschaftlicher Tatsachen in der Syphilisaufklärung. $>$ Die Schiffbrüchigen im Deutschen Theater zu Berlin (1913), in: «Der Hautarzt», Jg. LIII, H. 4, 2002, S. 268-276.

${ }^{46}$ L. FLECK, Entstehung und Entwicklung einer wissenschaftlichen Tatsache, a.a.O., S. 63.

${ }^{47}$ L. FLECK, Denkstile und Tatsachen, a.a.O., S. 232; L. FLECK, Entstehung und Entwicklung einer wissenschaftlichen Tatsache, a.a.O., S. 140.

${ }^{48}$ H.J. SANDKÜHLER, Kritik der Repräsentation, a.a.O., S. 208.

${ }^{49}$ L. FLECK, Denkstile und Tatsachen, a.a.O., S. 354. 